\title{
Measurement of groove features and dimensions of the vertical test cathode and the choke joint of the superconducting electron gun cavity of the Energy Recovery LINAC
}

\section{Hammons \& M. Ke}

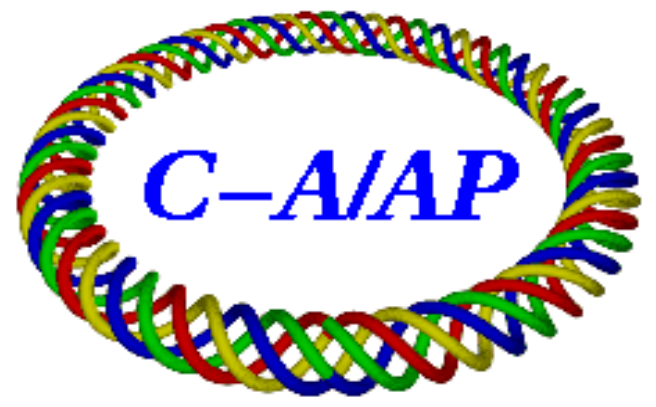

\section{Collider-Accelerator Department Brookhaven National Laboratory Upton, NY 11973}

Notice: This document has been authorized by employees of Brookhaven Science Associates, LLC under Contract No. DE-AC02-98CH10886 with the U.S. Department of En ergy. The United States Government retains a nonexclusive, paid-up, irrevocable, world-wide license to publish or reproduce the published form of this document, or allow others to do so, for United States Government purposes. 


\title{
Measurement of Groove Features and Dimensions of the Vertical Test Cathode and the Choke Joint of the Superconducting Electron Gun Cavity of the Energy Recovery LINAC
}

\author{
Lee Hammons, Ming Ke
}

\section{Introduction}

A testing program for the superconducting electron gun cavity that has been designed for the Energy Recovery LINAC is being planned. The goal of the testing program is to characterize the RF properties of the gun cavity at superconducting temperatures and, in particular, to study multipacting that is suspected to be occurring in the choke joint of the cavity where the vertical test cathode is inserted. The testing program will seek to understand the nature and cause of this multipacting and attempt to eliminate it, if possible, by supplying sufficient voltage to the cavity.

These efforts are motivated by the multipacting issues that have been observed in the processing of the fine-grain niobium gun cavity. This cavity, which is being processed at Thomas Jefferson National Laboratory for Brookhaven, has encountered multipacting at a gradient of approximately $3 \mathrm{MV} / \mathrm{m}$ and, to date, has resisted efforts at elimination. Because of this problem, a testing program is being established here in $\mathrm{C}-\mathrm{AD}$ that will use the large-grain niobium gun cavity that currently resides at Brookhaven and has been used for room-temperature measurements. The large-grain and fine-cavities are identical in every aspect of construction and only differ in niobium grain size. Thus, it is believed that testing and conditioning of the large-grain cavity should yield important insights about the fine-grain cavity.

One element of this testing program involves characterizing the physical features of the choke joint of the cavity where the multipacting is believed to be occurring and, in particular the grooves of the joint. The configuration of the cavity and the vertical test cathode is shown in Figure 1. In addition, it is important to characterize the groove of the vertical test cathode. The grooved nature of these two components was specifically designed to prevent multipacting. However, it is suspected that, because of the chemical processing that the fine-grain gun cavity underwent along with the vertical test cathode, the geometry of these grooves was altered, presenting the possibility that multipacting may, in fact, be occurring in this area and contributing to the low gradients that have been observed in the fine-grain cavity. 
Therefore, the Survey and Alignment group in C-AD engaged in measurements of the cavity joint, shown in Figure 2 and the cathode weldment, shown in Figure 3 for the purpose of characterizing the grooves in both the cavity and the vertical test cathode and comparing the dimensions of the cathode with those of the prints supplied by Advanced Energy Systems (AES), the original designer and manufacturer of both the test cathode and the electron gun cavity, in preparation to have a new one manufactured. The goal was to ensure that the articles as built matched the design prints in preparation for manufacturing a new vertical test cathode. This report describes the data collected by the Survey group in these efforts. The endeavor was challenging for the group given the millimeter-scale dimensions of the grooves and the requirement for high precision.

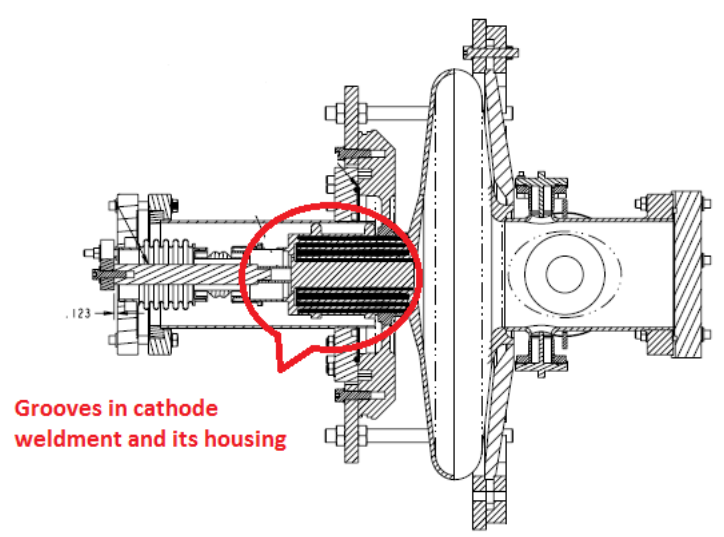

Figure 1: The assembly of the cathode weldment and its housing.

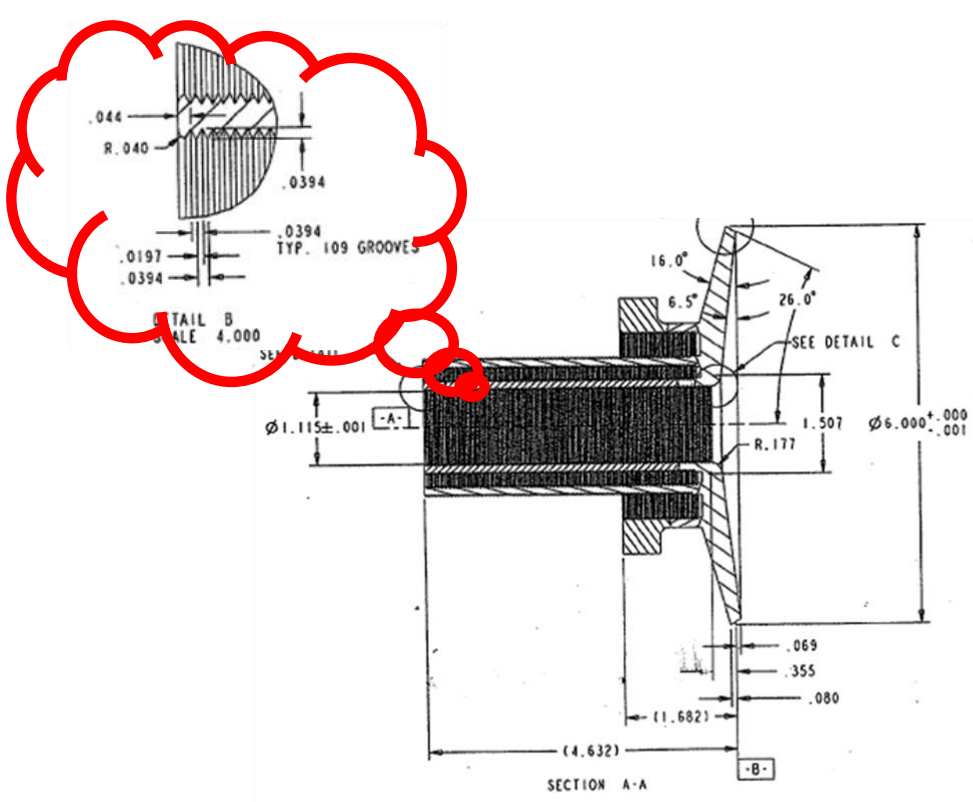

Figure 2: Dimensions of the cavity choke joint (dimensions in inches). 


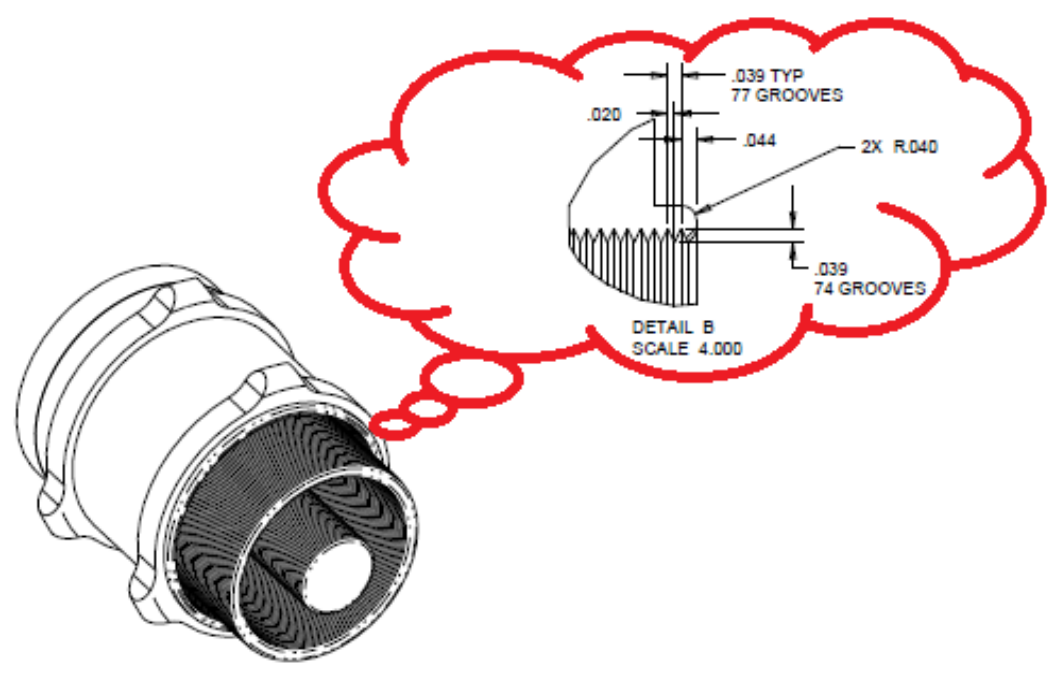

Figure 3: Dimensions of the grooves for the cathode weldment (in inches).

\section{Methodology}

During the process of measurement, traditional alignment telescope and modern techniques such as 1D laser distance measurement and digital camera imaging were all employed. The chief focus of the measurements was on the grooves, and an R\&D program was conducted. Ultimately, a feasible, combined approach was adopted that not only accomplished the specific goals of the measurement effort but also enriched the Survey group's toolbox and enhanced the group's capabilities.

It is important to note that because only a portion of the grooved surface was accessible on either the cavity or the cathode, only a sample of the grooves on these components could be measured by any of the methods outlined below. In general, an attempt was made to measure as much of the exposed or accessible surface as possible. Figures 4 and 5 show the groove orientation and terminology used throughout the study.

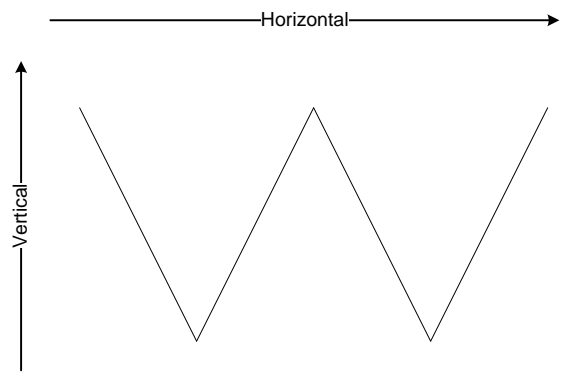

Figure 4: Schematic showing groove orientation. 


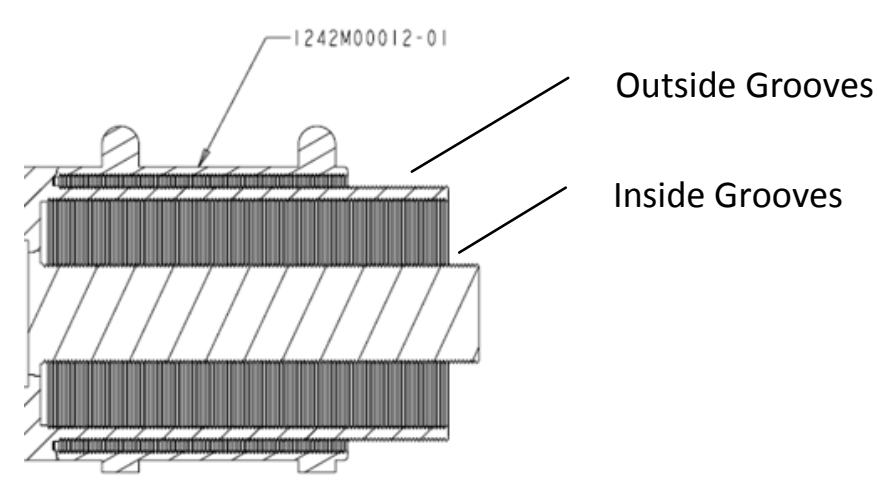

Figure 5: Schematic showing measurement areas of cathode.

In addition, measurements were made of the gross dimensions of certain features of the cathode to verify that the structural features of the cathode were the same as those indicated on the print. These measurements were made using traditional methods, and were necessary in order to assure that the prints matched the as-built component. The results are discussed below.

Four methods of measurement were applied to the grooves:

- 1-D laser distance measurement for dimension measurement in the elevation direction;

- Alignment telescope

- Digital camera measurement, and;

- Articulated arm. This technique only was used on the choke joint of the cavity with $0.3 \mathrm{~mm}$, $0.5 \mathrm{~mm}$, and $0.7 \mathrm{~mm}$ probes.

\section{1-D Laser Distance Measurement}

Due to the difficulty of aiming at the valleys of each of the grooves by the alignment telescope, the 1D laser distance measurement was deemed as the potential method to measure the vertical dimension although the alignment telescope can also measure the vertical dimension. The laser is a non-contact device, and during the test, the laser emitter was fixed on a moveable base with a micrometer, allowing the laser to move in $0.01 \mathrm{~mm}$ intervals parallel to the axis of the measurand.

\section{Alignment Telescope}

The alignment telescope was mainly used to measure the interval in the horizontal direction between grooves. The smallest division is equal to 0.001 inches displacement indicated by the telescope's micrometer and allows precise reading between grooves. In measuring the cavity, it proved convenient to set the height of the alignment telescope to aim at the lowest part of the 
measurand. The telescope was also used for measuring the vertical dimension in the aggregate between the highest line and the lowest line similar to the method described below for the digital camera.

\section{Digital Camera}

The camera was convenient for getting clear pictures of the grooves. A key factor in the use of the camera is establishing the correct scale of the images. To this end, a steel ruler was used and incorporated into pictures as shown in Figure 6. The cathode weldment was rotated every $45^{\circ}$ to get a complete presentation. The lighting was also found to be important to get a desirable picture. Dozens of pictures had to be taken to tune the effect of lighting.

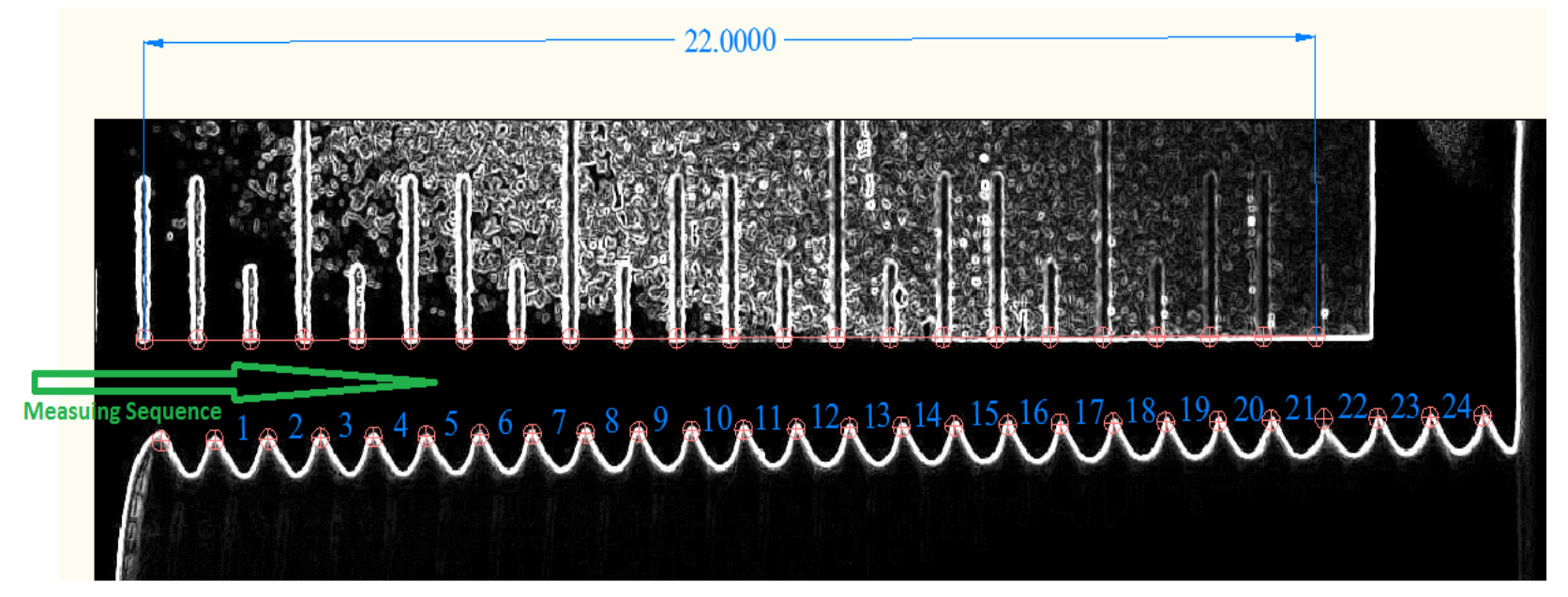

Figure 6: Image collected by digital camera after processing using ImageJ software. The ruler and the exposed end of the cathode stalk are visible. Note that each groove except for the groove at the left end of the image is numbered.

When analyzing the picture, two kinds of software were employed: a Java program called ImageJ was used to pretreat the picture. This software provided a means of clearly delineating all of the edges in the picture. AutoCAD was then used to further analyze the image and obtain the dimensions of interest. From here, several more steps were required:

1. The image of the measurand and the ruler were set to the same scale.

2. A line was passed through points of the same vertical dimension, at or near the peak of each groove, in order to provide a reference line for measurement of the horizontal dimension of each groove. This allowed for a consistent measure of the horizontal dimension of each groove.

3. Two parallel lines through the highest peak and the lowest point were set to get the amplitude in the vertical direction. 
4. Finally, the distances were measured and plotted to show the variation from the nominal design value.

Measurement of the cavity choke joint using this method was complicated by the mounting arrangements that were required for the cavity relative to the camera. Due to the significant difference in elevation between the camera and the portion of the cavity to be measured, the camera was tilted to its maximum angle by using an adjustment screw, and the face of the ruler was set perpendicularly to the optical axis of the camera. It also important to note that only the outer surface of the choke joint could be measured using any of the methods.

\section{Articulated Arm}

The articulated arm is a direct measurement with extremely small probes of $0.3 \mathrm{~mm}, 0.5 \mathrm{~mm}$, and $0.7 \mathrm{~mm}$ width applied to the surface of the measurand. This method was used only for measurement of the cavity choke joint. Measurement using the arm proved problematic: indeed, four probes were broken because the probes are rather brittle, and it was difficult to control the pressure applied to the measurand.

Also, time for these measurements was rather limited. Despite these difficulties, five to six points around the circumference of each groove were measured and distances were calculated using the best-fit line constructed from the centers of the circles formed by the series of measurements to each point using spatial analysis software as shown in Figure 7. Because the articulated arm cannot precisely measure the peaks or valleys of the grooves, it could not be used to measure the vertical dimension of the grooves.

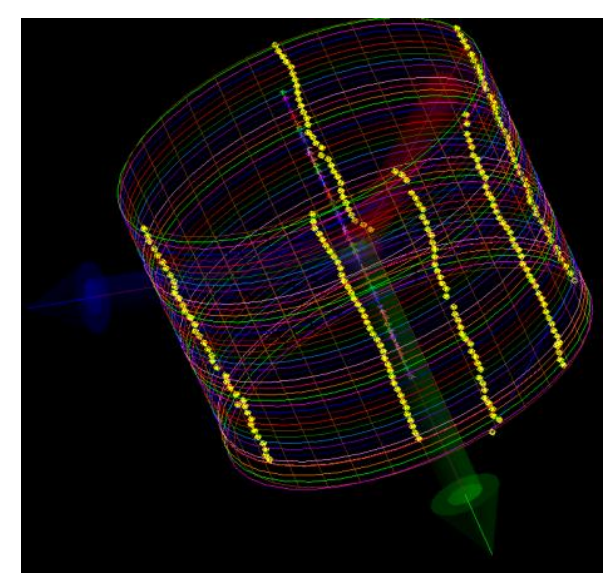

Figure 7: The constructed circles were constructed from measurements made using the articulated arm. 


\title{
Results
}

\section{1-D Laser Distance Measurement}

Several attempts using the laser were unsuccessful in measuring the dimensions of the grooves. A typical result is shown in Figure 8. The result shows sharp mutations in the measured groove structure throughout. Furthermore, the amplitude is significantly different than the nominal design value. The failure of this approach is likely attributable to the shiny surface of the measurand, scattering light to such a degree that it is difficult to make proper measurements.

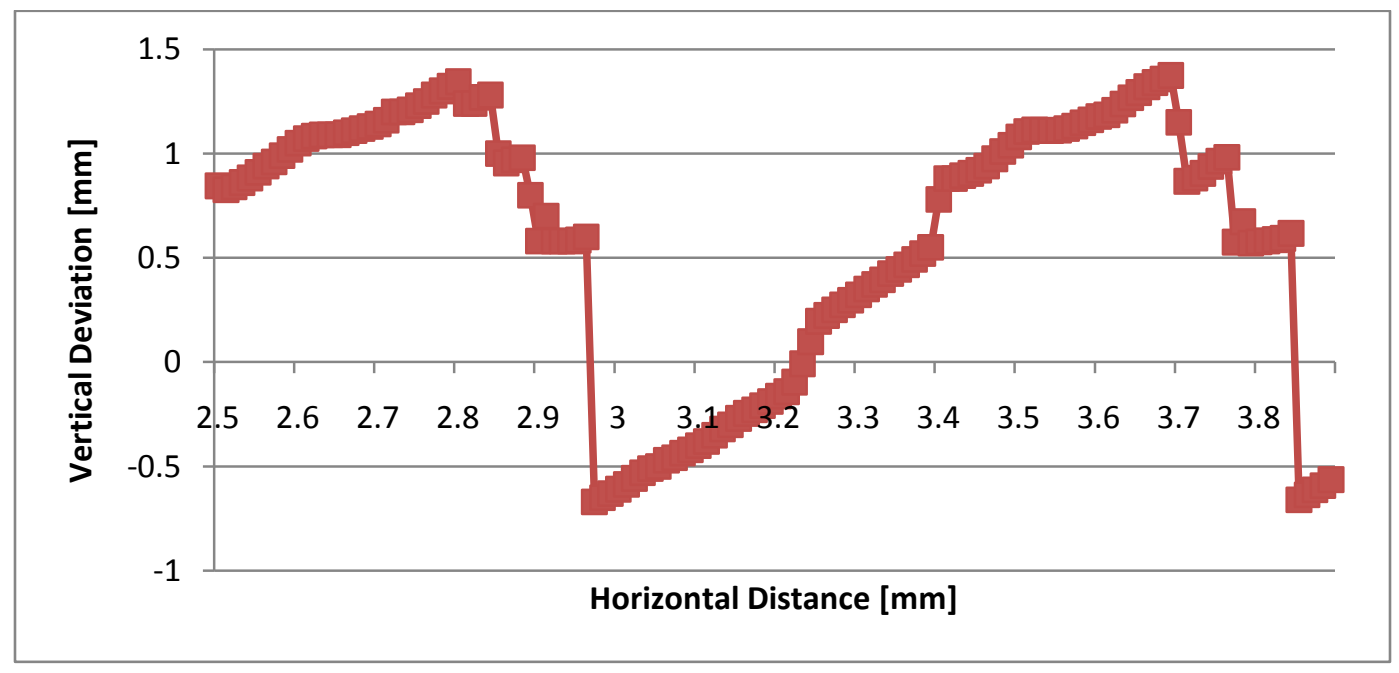

Figure 8: Typical 1-D laser distance measurement result.

\begin{abstract}
Alignment Scope
The data from the optical alignment scope are heavily dependent on the visual acuity of the observer and, therefore, only two groups of data at 0 degrees and 45 degrees were used to calculate the distance between the peaks. The data are shown in Figures 12 and 13 for the outside grooves and Figures 23 and 24 for the inside grooves. The deviations seem to be fairly well distributed about the nominal value, particularly for the 0 -degree measurement. However, the 45 -degree measurement shows a marked tendency toward positive variations. Given the statistical distribution of the 0 -degree result, some systematic error is suggested in the measurement. One possible source of error is in the nature of the rotation of the cathode - if the cathode alignment changes through the rotation, systematic errors may result. Note that this is distinct from the measurement that might be expected after alteration of the geometry due to chemical etching. As suggested in Figure 9, etching would tend to round the peaks and valleys of the grooves without
\end{abstract}


necessarily altering the distance between peaks and valleys. Etching would be expected only to alter the amplitude of the grooves. Thus, a measurement of the grooves should, under ordinary circumstances, still yield statistical variations.

Vertical meausurement of the grooves was taken in the aggregate by measuring the distance from the highest line to the lowest line for the outside grooves in Table 1. No vertical measurements for the inside grooves were performed for the inner grooves.

Because of the difficulties of the measurement as suggested above, the results are deemed somewhat less reiliable than the measurements using the camera. Nonetheless, the results are marked different from the nominal design value of $0.9906 \mathrm{~mm}$, showing a 7 - 13\% variation from nominal. Measurement of the vertical dimension for the inside grooves was not conducted using the alignment scope.

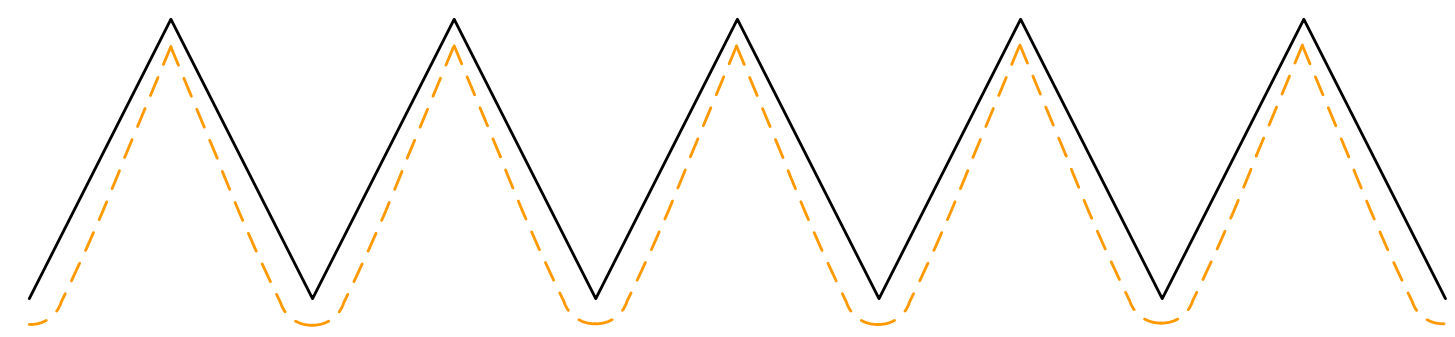

Figure 9: Schematic showing possible effect of chemical etching on groove structure. This cartoon is greatly exaggerated, but suggests that groove amplitude may be altered but not horizontal spacing for a given etch depth.

Table 1: Vertical Dimension Measurements at Various Rotations for Cathode Outer Grooves

\begin{tabular}{ccc}
\hline \multirow{2}{*}{ Rotation [deg] } & \multicolumn{2}{c}{ Distance [mm] } \\
\cline { 2 - 3 } & Telescope & Camera \\
\hline 0 & 0.86360 & 0.8313 \\
45 & 0.86868 & 0.8140 \\
90 & 0.85852 & 0.8335 \\
135 & 0.86868 & 0.8390 \\
180 & 0.86360 & 0.8605 \\
225 & 0.89408 & 0.9250 \\
270 & 0.90932 & 0.9131 \\
315 & 0.92710 & 0.9085 \\
360 & 0.85852 & 0.8471 \\
\hline Average & 0.87579 & 0.8636 \\
Std. Dev. & 0.02356 & 0.0411 \\
\hline
\end{tabular}


Data for the cavity choke joint using this technique appears in Figure 46 for a single orientation only. It was unexpectedly difficult to properly aim the scope at the measurand and make a measurement. Therefore, the data collected using this technique must be viewed with suspicion. The data shows a very small maximum deviation ( $\sim-0.06 \mathrm{~mm}$ or $6 \%)$ but a majority of positive deviations from the nominal values. Measurement of the vertical dimension on the choke joint was taken by measuring the distance from the lowest point to the highest point along the grooves and yielded a value of $0.83058 \mathrm{~mm}$.

Table 2: Vertical Dimension Meaurements at Various Rotations for Cathode Inner Grooves using Camera

\begin{tabular}{cc}
\hline Rotation [deg] & Distance $[\mathrm{mm}]$ \\
\hline 0 & 0.5987 \\
45 & 0.6817 \\
90 & 0.6892 \\
135 & 0.6842 \\
180 & 0.5975 \\
225 & 0.6512 \\
270 & 0.6050 \\
315 & 0.6304 \\
360 & 0.5699 \\
\hline Average & 0.6342 \\
Std. Dev. & 0.0443 \\
\hline
\end{tabular}

\section{Digital Camera}

The digital camera data for measurements of the outside grooves of the vertical test cathode are shown in Figure 15 through 23. Data for the inside grooves are shown in 23 through 43 . All of the figures show the variations from the nominal $0.9906 \mathrm{~mm}$ design value. The charts demonstrate that the deviation along the horizontal direction is less than $0.024 \mathrm{~mm}$ or $2 \%$ between grooves regardless of the orientation. The measuring sequence is shown in Figure 6 for both the inside and outside grooves.

The distances from the highest line to the lowest line along the vertical direction of the cathode are listed in Table 1 for outside grooves and Table 2 for inside grooves. For outside grooves, the digital camera measurement changes from $0.8140 \mathrm{~mm}$ to $0.9250 \mathrm{~mm}$. Compared with the nominal value of $0.9906 \mathrm{~mm}$, the data indicates that there is a deviation of between 7 and $18 \%$ - a significant variation. For the inside grooves, the measured deviation is even larger, with measurements less than $0.7 \mathrm{~mm}$ or greater than $30-43 \%$ deviation from the nominal design value. 
For the grooves of the cavity choke joint, calculated measurements of the horizontal spacing between grooves using AutoCAD are shown in Figure 45. The vertical dimension of the cavity was measured in a matter similar to the above for the cathode and yielded a distance between the upper and lower lines formed by the peaks and valleys of the grooves of $0.8322 \mathrm{~mm}$.

\section{Articulated Arm}

Data for the articulated arm measurements appears in Figure 44. It is clear that this data shows consistent and pronounced positive deviations from the nominal design value for the horizontal spacing. Given the limitations and difficulties of using the probe as discussed above, this data must be viewed with skepticism. However, it is consistent with data collected using other methods in that it consistently indicates larger horizontal spacing than the nominal value.

\section{Comparison of Prints to Solid Model}

Prompted by the discrepancies that were found in the vertical dimensions between the grooves on the cavity and the design prints, C. Cullen, the design engineer for the testing program, also inspected the ProE solid model of the cavity to determine if there were discrepancies. Both the prints and the solid model were supplied by AES. He found that while the majority of grooves on the cavity in the solid model matched the print design, the depth of the grooves situated on the outer annulus of the joint did not match the print design. The discrepancy is indicated below in Figure 10. The different is small, but significant given that all of the groove dimensions should be the same. Since it is impossible to verify the dimensions on the actual cavity in this location, it is not possible to determine whether the difference between the print and the solid model is actually found in the cavity or merely a flaw in the design files.

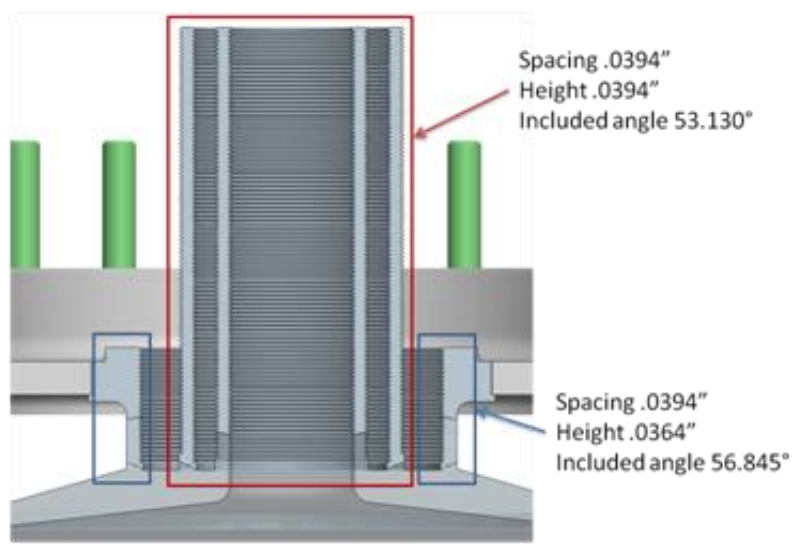

Figure 10: Dimensions found in the ProE solid model. Note that the areas that are surrounded by the blue box have grooves of a different depth (0.0364") than those found in the AES print (0.0394"). 


\section{Auxillary Measurements}

In addition to the measurements of the grooves, several other dimensions were checked in order to verify the overall dimensions of the vertical test cathode. The dimensions are identified in Figure 11 and were checked using the articulated arm. Measurements were taken three times, averaged, and compared with the design values from the print. The data is summarized in Table 3.

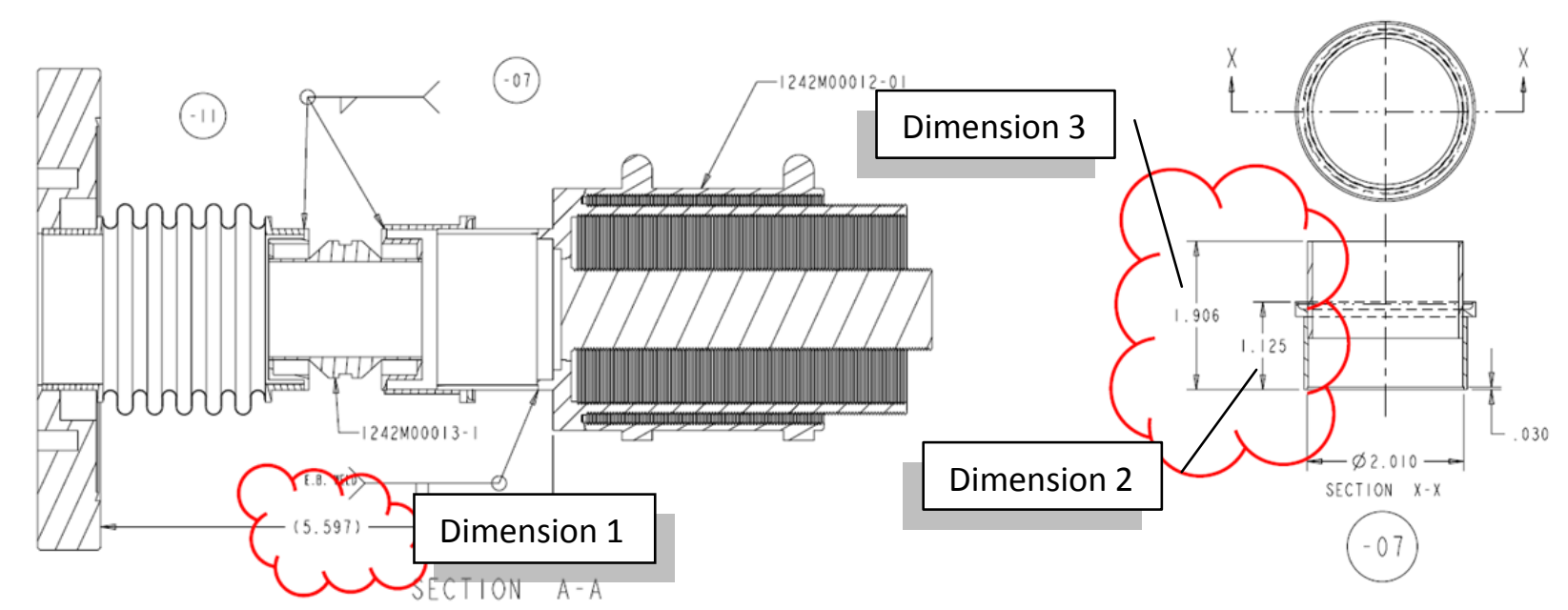

Figure 11: Gross dimensions of the vertical test cathode that were verified using the articulated arm. Dimensions in inches.

Table 3: Measurements of Gross Dimensions of Vertical Test Cathode (All measurements in millimeters)

\begin{tabular}{|c|c|c|c|c|c|c|}
\hline \multirow{2}{*}{ Dimension } & \multicolumn{3}{|c|}{ Measurement } & \multirow{2}{*}{ Average } & \multirow{2}{*}{ Design } & \multirow{2}{*}{ Deviation } \\
\hline & $1 \mathrm{st}$ & 2nd & $3 \mathrm{rd}$ & & & \\
\hline 1 & 136.1023 & 136.0919 & 136.1243 & 136.1062 & 142.1638 & -6.0576 \\
\hline 2 & 29.0374 & 29.0348 & 29.0464 & 29.0395 & 28.5750 & 0.4645 \\
\hline 3 & 53.3437 & 53.3683 & 53.3844 & 53.3655 & 48.4124 & 4.9531 \\
\hline
\end{tabular}

\section{Conclusions}

The measurements using the various techniques show that the geometry of the actual cathode differs from the AES prints. The most significant difference appears to be in the vertical dimension where deviations are on the order of 7 - 18\% for the outer grooves of the test cathode, depending on the measurement method, and $31-42 \%$ for the inside grooves. It is important to keep in mind that only a small portion of the grooves could be measured given the geometry of the cathode. 
Nonetheless, the differences are significant and are expected to be found for all the grooves. Not only are the deviations large but, as the camera images show, the valleys appear to be rounded, differing considerably from the sharp peaks and valleys indicated in the design. The horizontal dimension shows much smaller deviations with a maximum of $\sim 3 \%$ from the design value. There is some indication in this data of systematic error given the distribution of the data. It is suspected that this may be caused by small changes in orientation with rotation of the measurand.

Nonetheless, for the most part, the data shows only small deviations from the nominal design value in the horizontal spacing of the grooves on the cathode.

The cause of the changes in the vertical dimension from the design are suspected to have occurred due to chemical etching that may have occurred during the initial processing of the fine-grain cavity that included the processing of the vertical test cathode. Notice that the largest changes occur on the inner grooves of the cathode, consistent with the expectation that this portion of the cathode is most exposed to chemistry. The details of the processing history were not fully explored in this study, and it would be interesting to understand this history more fully, but the implication is that exposure of the grooves to chemistry has the potential to significantly alter their geometry in a manner which is apparently non-linear given the images obtained from study with the camera and which may change their RF characteristics. It would seem that caution should be exercised in the processing of these areas on subsequent articles.

As for the cavity, very little time was available to make a full study of the cavity, but the limited data suggests that the horizontal dimensions are likely within better than $\sim 4 \%(+/-0.02 \mathrm{~mm})$ of nominal design value. Limited vertical dimension data was obtained for the cavity using the camera and telescope techniques and these values are vary by $\sim 16 \%$ from the nominal value for both methods. Given that the large-grain cavity had not been exposed to chemical processing at the time of the measurement, the deviation seems large. However, it is also important to note that this measurement is an agregate measurement and is, in general, a less precise measure of the height of the grooves than the spacing between grooves. It is also of concern that the solid model file does not match the print file. Unfortunately, because of the location of the discrepancy in the design files, it is impossible to corroborate the dimensions with measurements. 


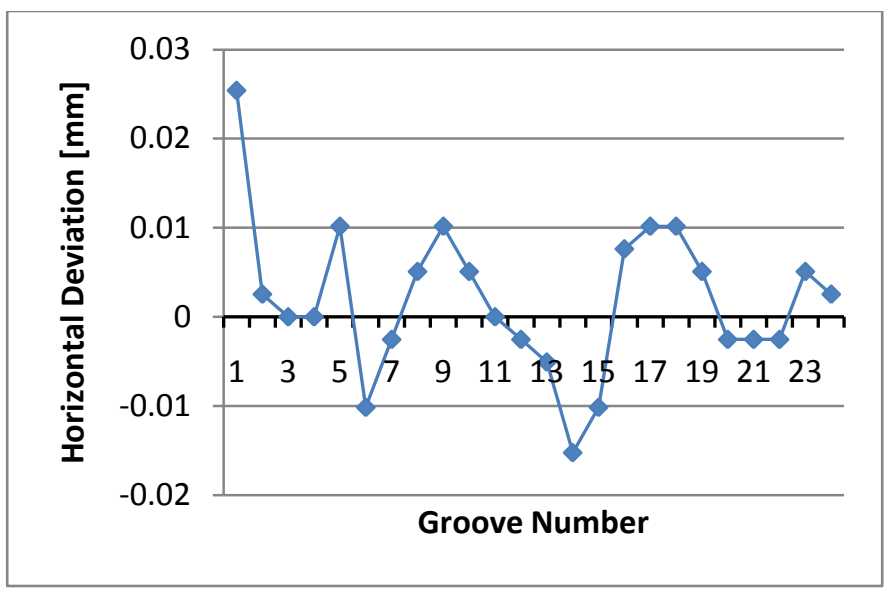

Figure 12: 0-degree orientation for outer grooves using alignment telescope.

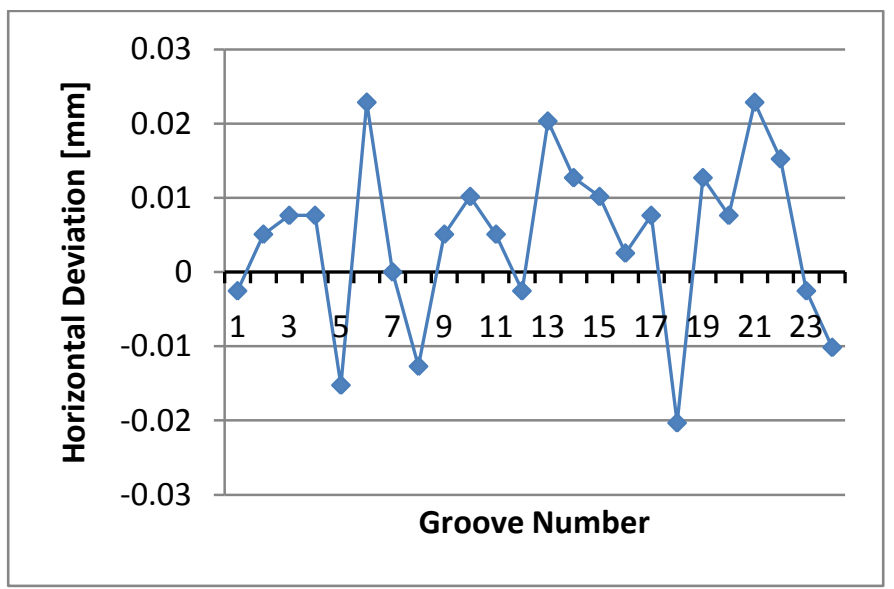

Figure 13: 45-degree orientation for outer grooves using alignment telescope.

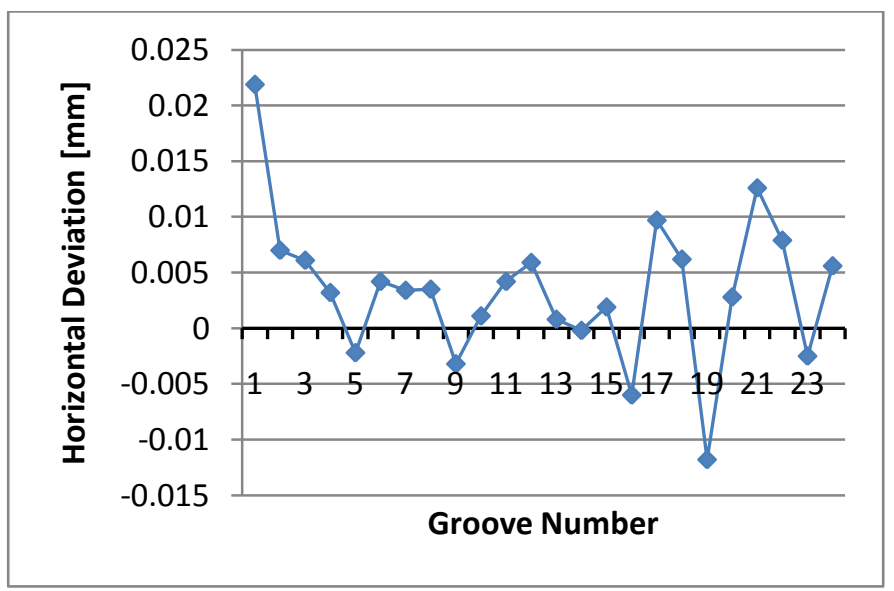

Figure 14: 0-degree orientation for outer grooves using camera.

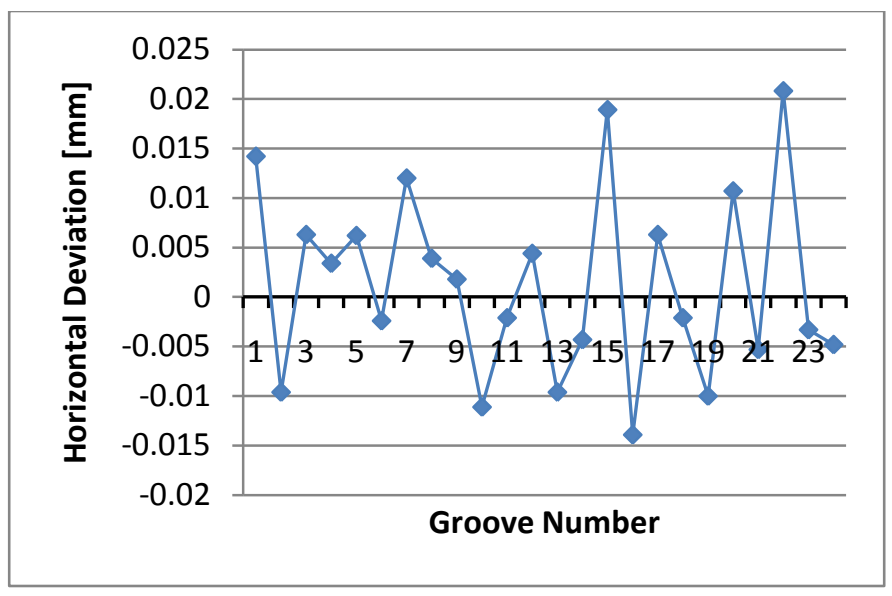

Figure 15: 45-degree orientation for outer grooves using camera.

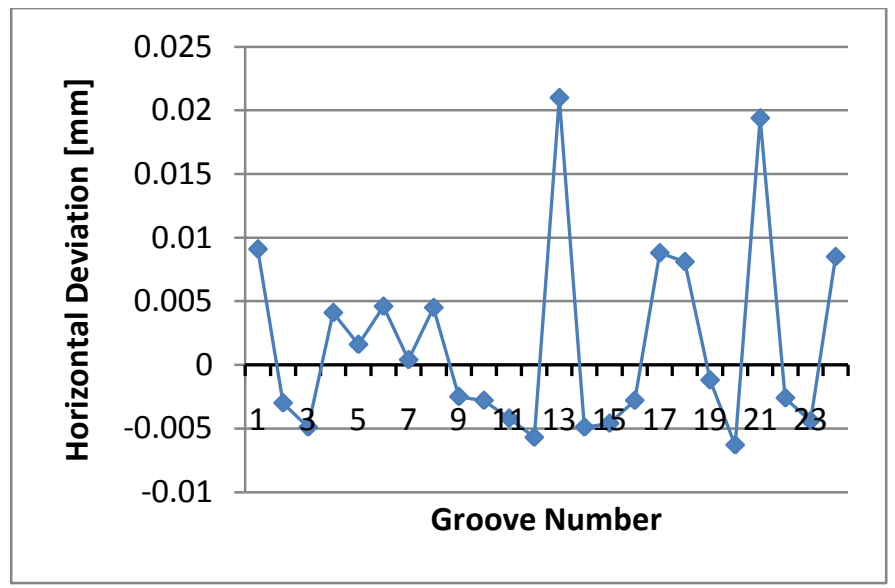

Figure 16: 90-degree orientation for outer grooves using camera.

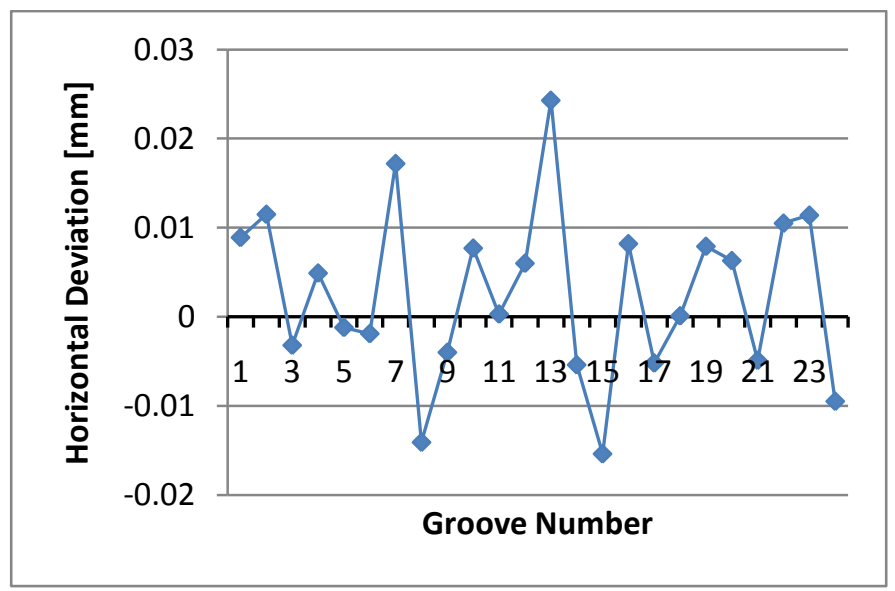

Figure 17: 135-degree orientation for outer grooves using camera. 


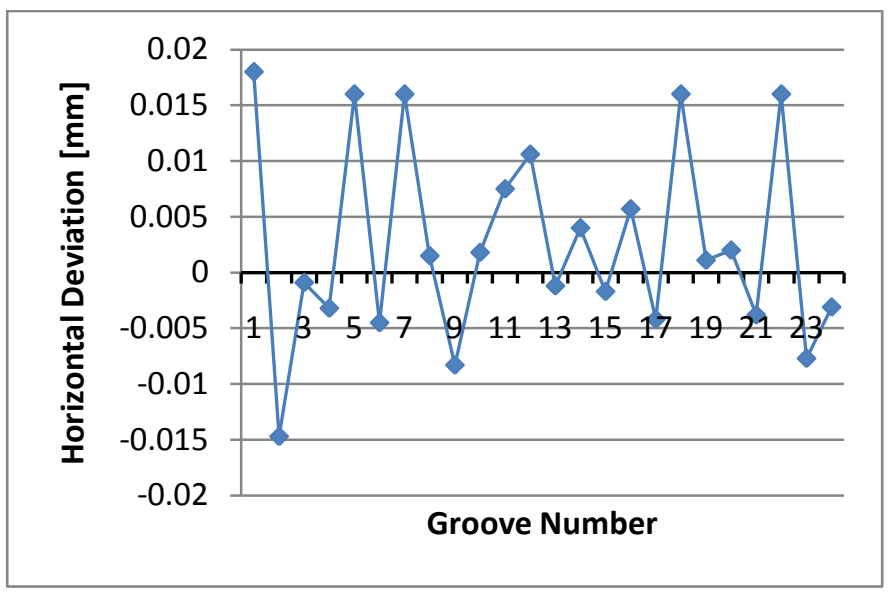

Figure 18: 180-degree orientation for outer grooves using camera.

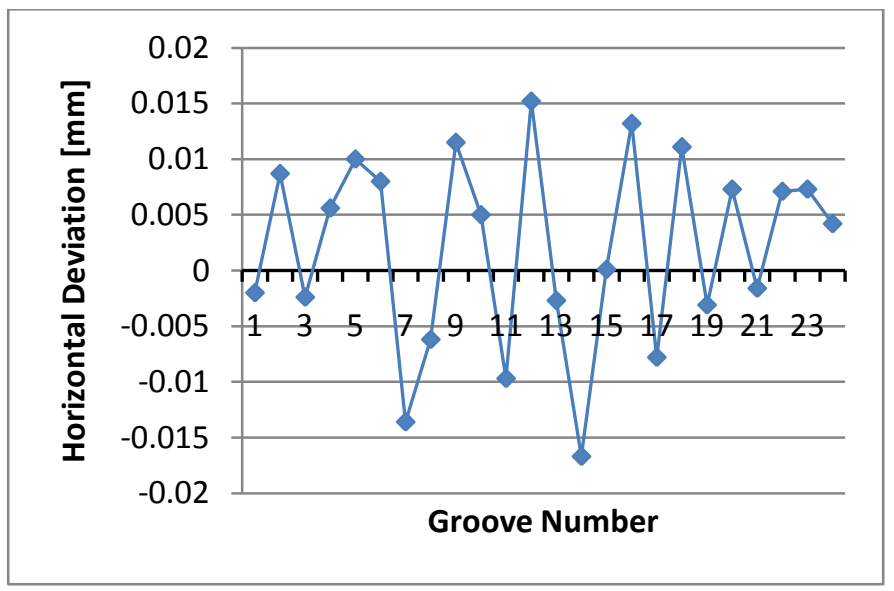

Figure 19: 225-degree orientation for outer grooves using camera.

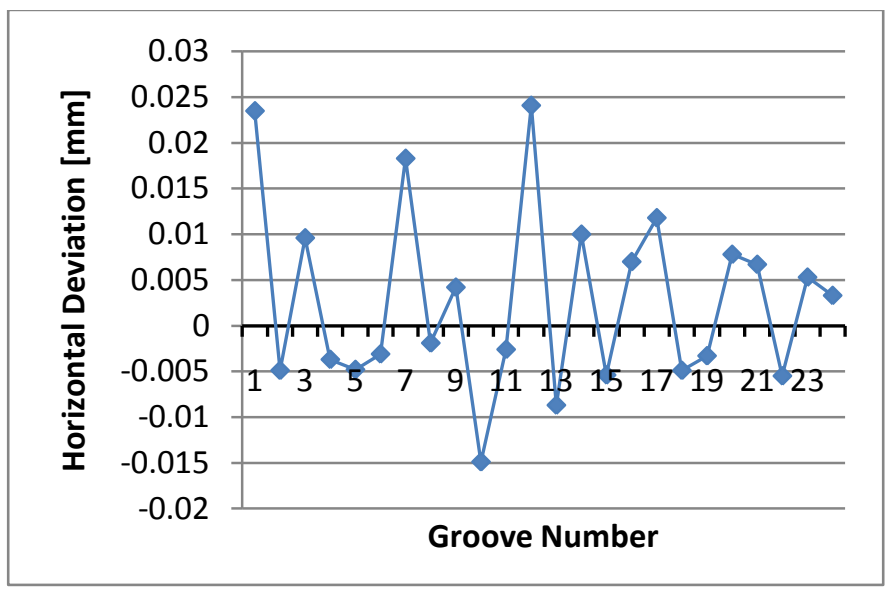

Figure 20: 270-degree orientation for outer grooves using camera.

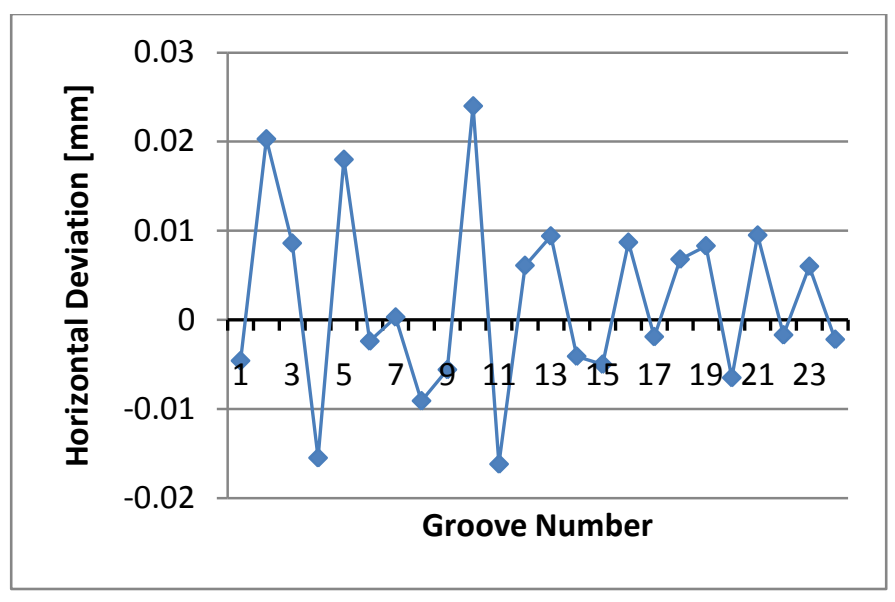

Figure 21: 315-degree orientation for outer grooves using camera.

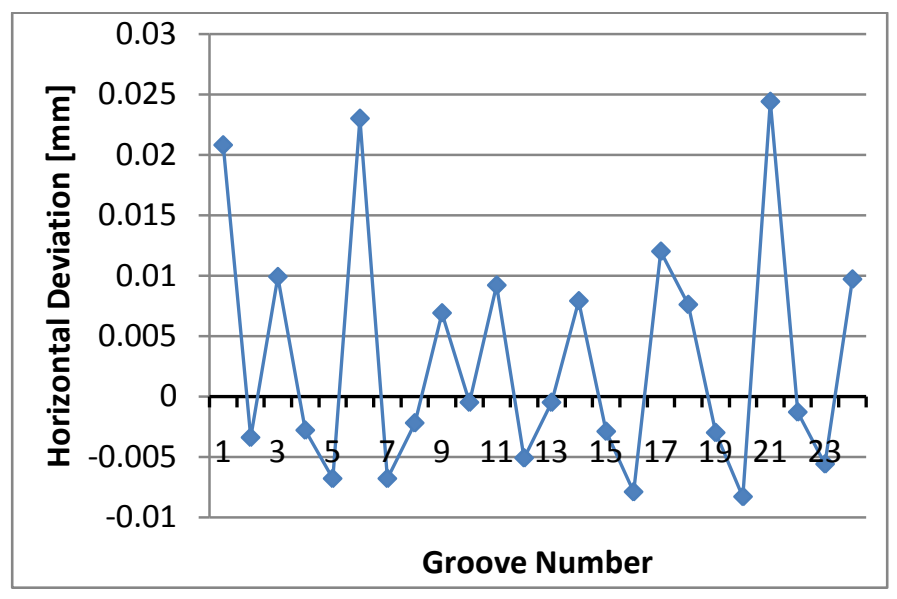

Figure 22: 360-degree orientation for outer grooves using camera.

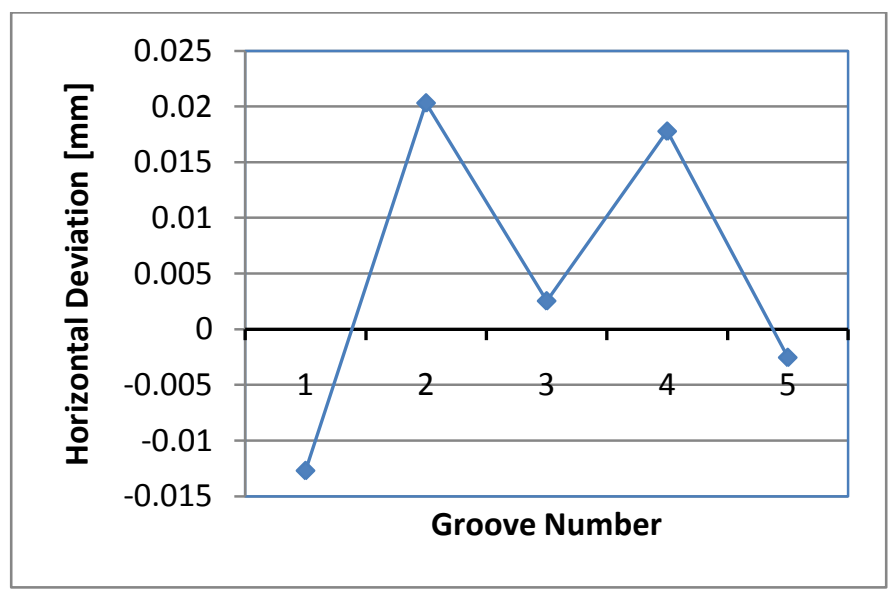

Figure 23: 0-degree orientation for inner grooves using alignment telescope. 


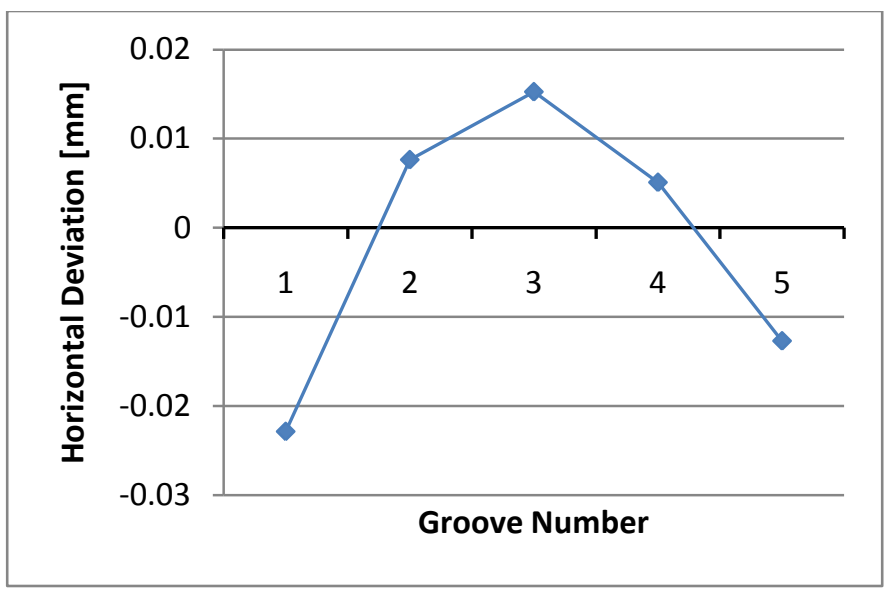

Figure 24: 45-degree orientation for inner grooves using alignment telescope.

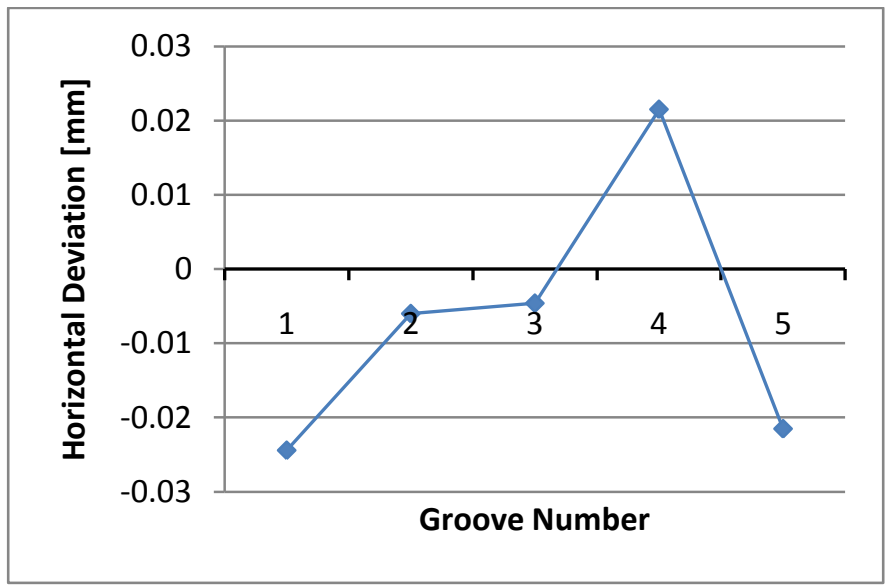

Figure 25: 0-degree orientation for inner grooves using camera.

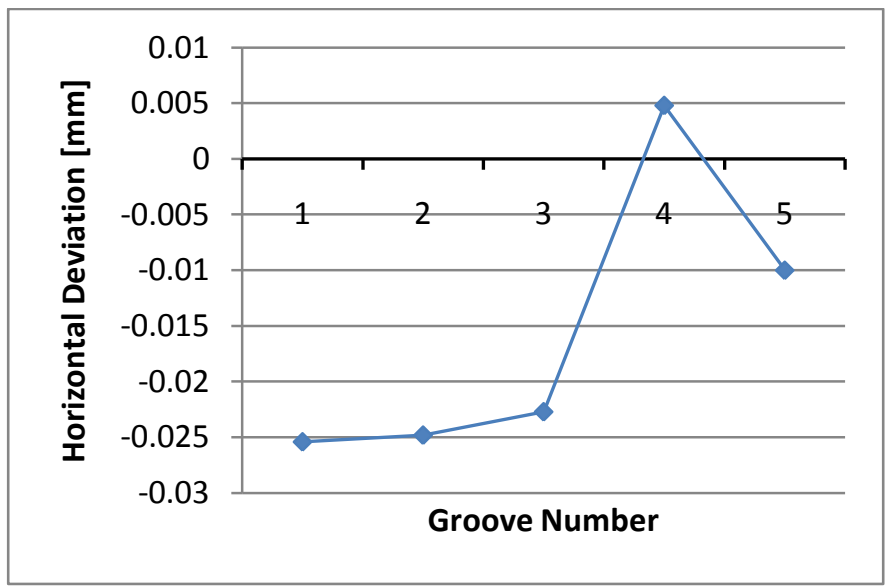

Figure 26: 45-degree orientation for inner grooves using camera.

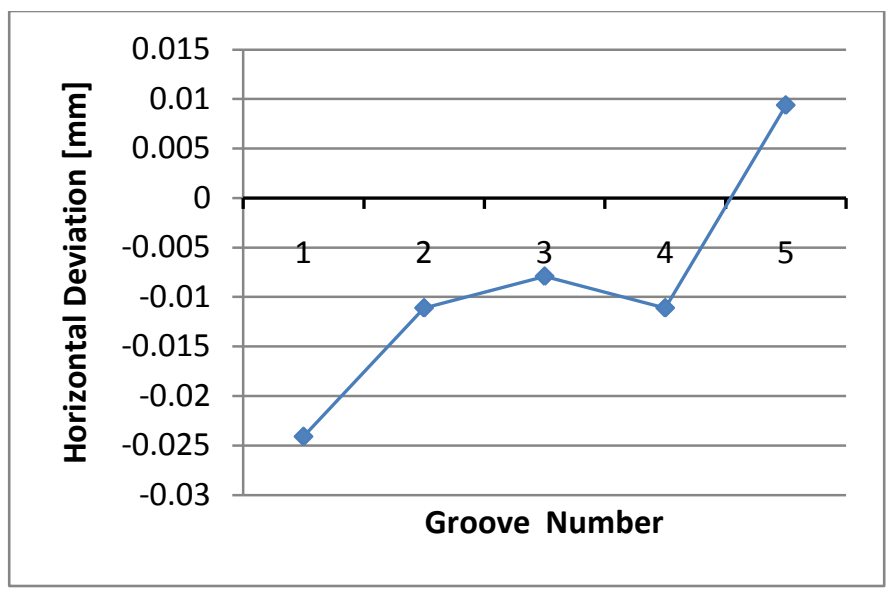

Figure 27: 90-degree orientation for inner grooves using camera.

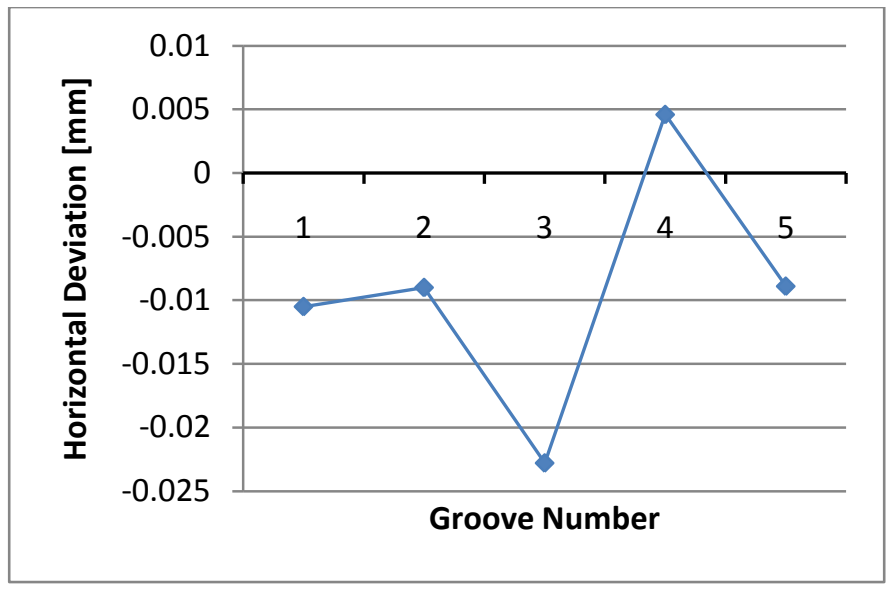

Figure 28:135-degree orientation for inner grooves using camera..

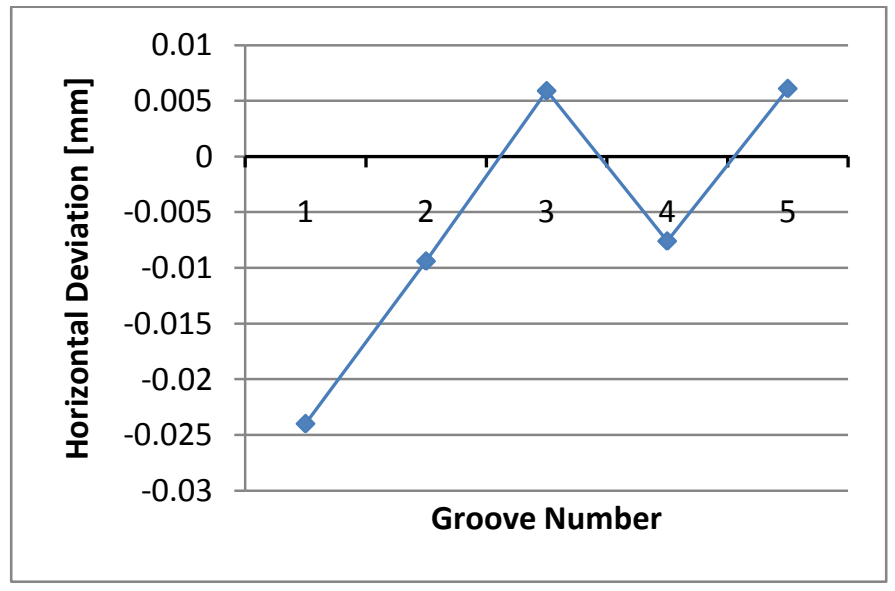

Figure 29: 180-degree orientation for inner grooves using camera. 


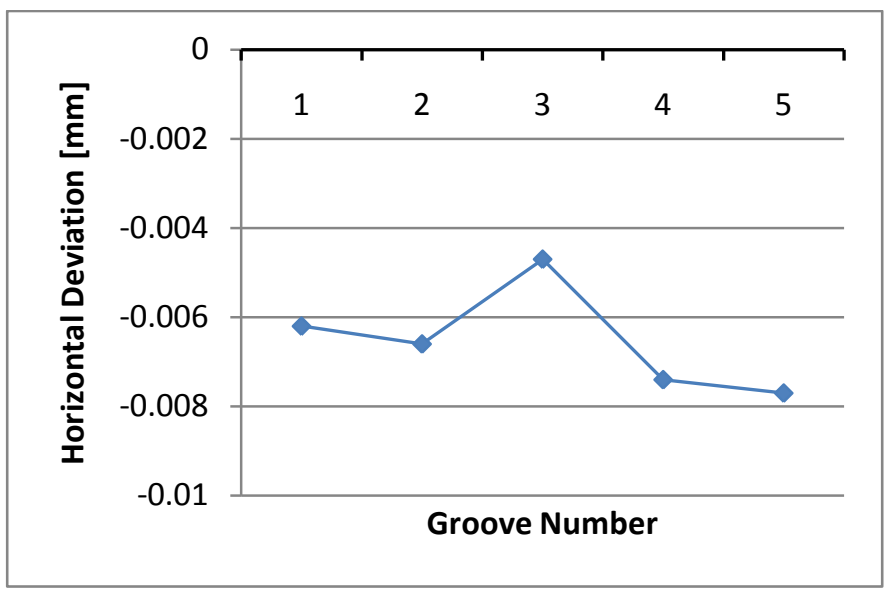

Figure 40: 225-degree orientation for inner grooves using camera.

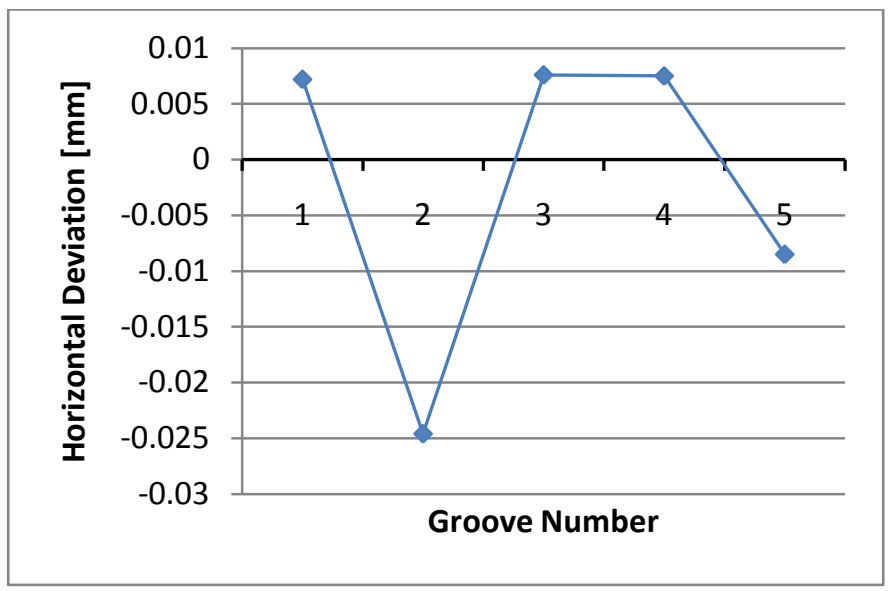

Figure 41:270-degree orientation for inner grooves using camera.

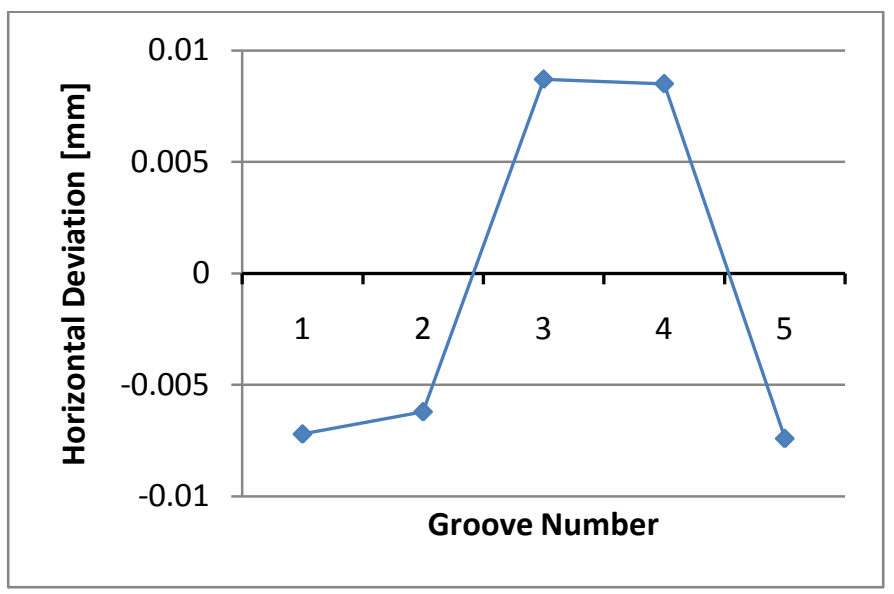

Figure 42: 315-degree orientation for inner grooves using camera.

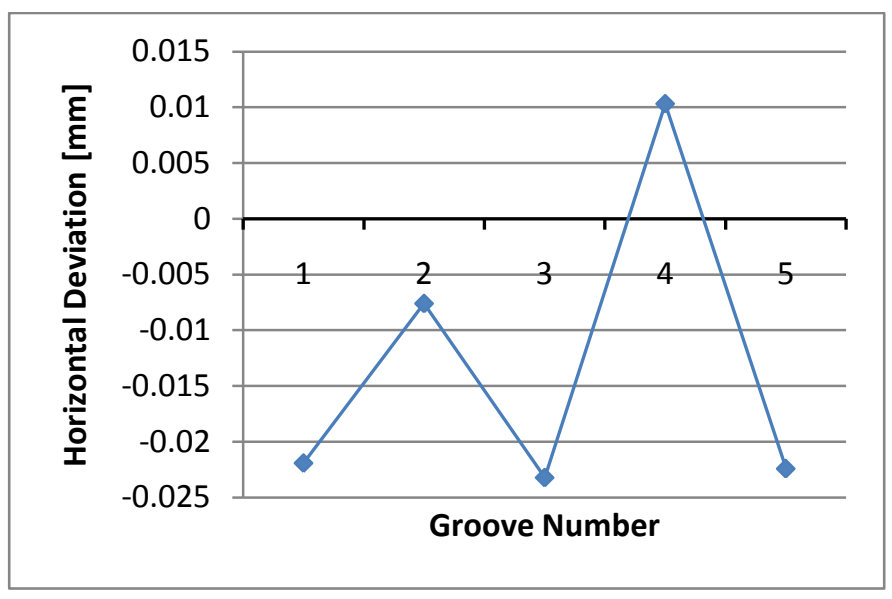

Figure 43: 360-degree orientation for inner grooves using camera.

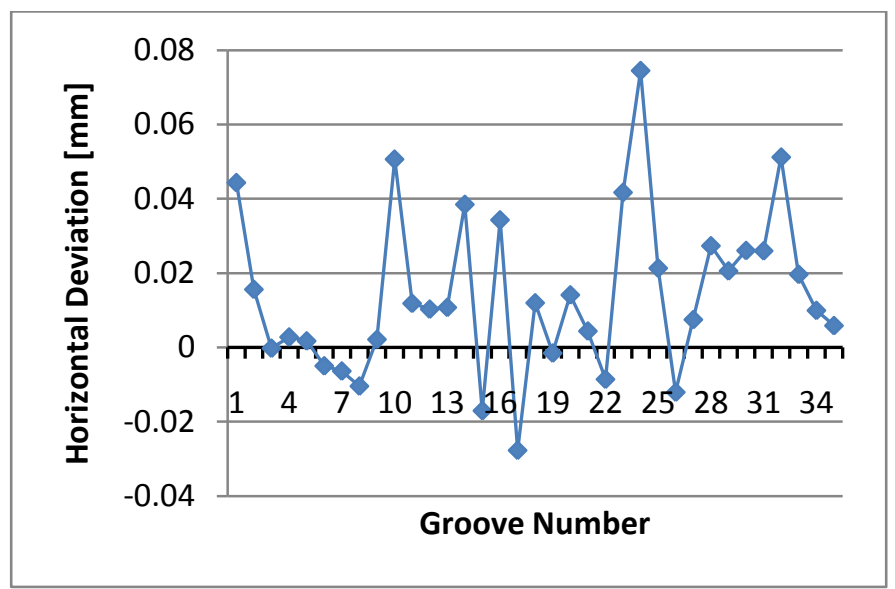

Figure 44: Deviations for cavity choke joint using articulated arm.

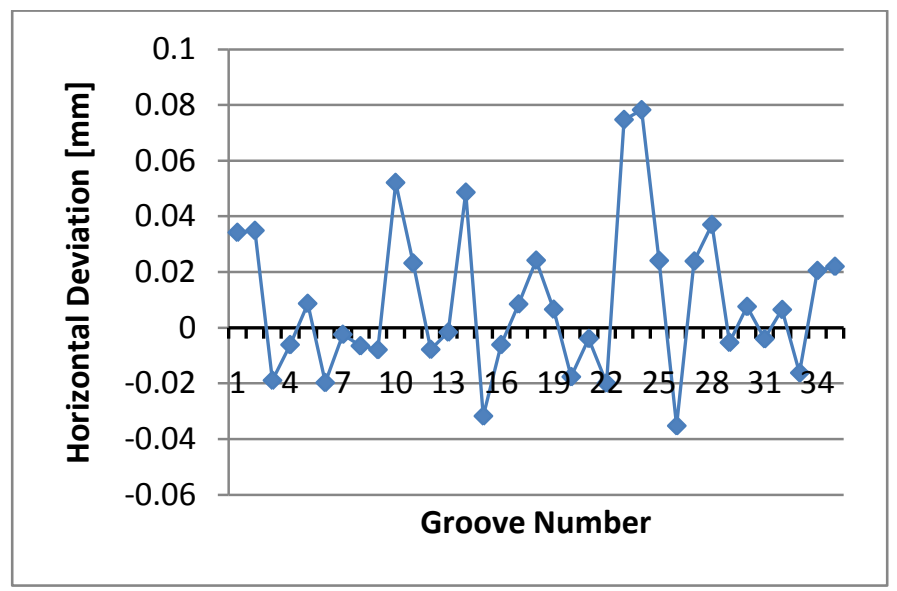

Figure 45: Deviations for cavity choke joint using camera. 


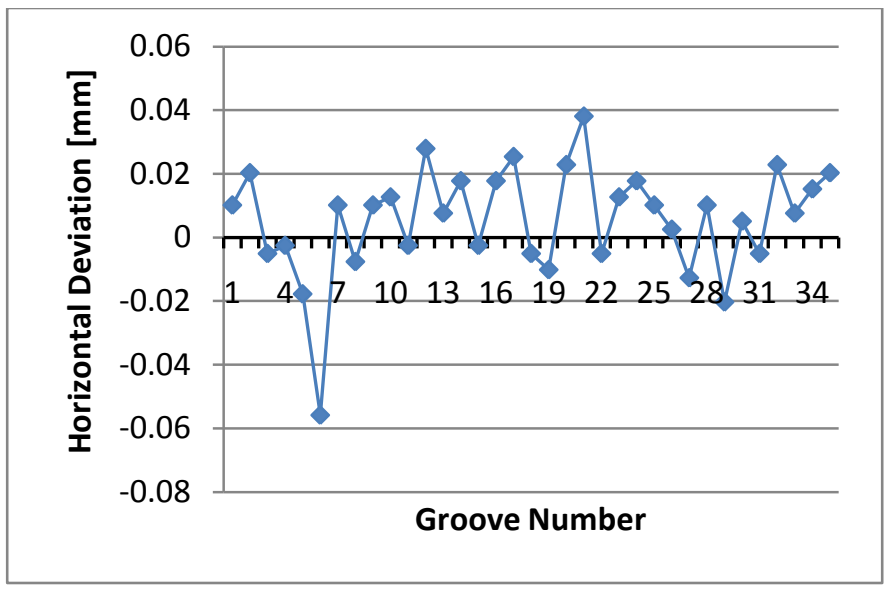

Figure 46: Deviations for cavity choke joint using alignment telescope. 